\title{
A note on $b_{f}$-spaces and on the distribution of the functor of the Dieudonné completion
}

https://doi.org/10.1515/taa-2021-0201

Received 6 December, 2021; accepted 29 December, 2021

Abstract: A subset $B$ of a space $X$ is said to be bounded (in $X$ ) if the restriction to $B$ of every real-valued continuous function on $X$ is bounded. A real-valued function on $X$ is called $b_{f}$-continuous if its restriction to each bounded subset of $X$ has a continuous extension to the whole space $X . b_{f}$-spaces are spaces such that $b_{f^{-}}$-continuous functions are continuous. We take advantage to the exponential map in the realm of $b_{f^{-}}$ spaces in order to study $b_{f}$-extensions of $b_{f}$-continuous functions. This allows us to improve several results concerning the distribution of the functor of the Dieudonné completion. We also prove that a relative version of the classical Glicksberg's theorem characterizing the product of two pseudocompact spaces is valid for $k_{r^{-}}$ spaces. In the last section we show that $b_{f}$-hemibounded groups are Moscow spaces and, consequently, they are strong- $P T$-groups.

Keywords: $b_{f}$-space, $b_{f}$-continuous function, exponential map, Dieudonné completion, $k_{r}$-space, Moscow space, strong- $P T$-group

MSC: 54C10, 54C20, 54C35, 54C45, 54D60, 54G99, 54H11

\section{Introduction}

Glicksberg in [16] showed an elegant theorem on the distribution of the functor $\beta$ of the Stone-Čech compactification reproved later by another method in [13] by Z. Frolík: For infinite spaces $X$ and $Y$, the equality $\beta(X \times Y)=\beta(X) \times \beta(Y)$ holds if and only if the product space $X \times Y$ is pseudocompact. The interest and importance of the Gliscksberg-Frolik theorem lies in the fact that the distribution of the functor $\beta$ is characterized by means of a topological property of the product space $X \times Y$.

The attempt of obtaining a similar result for the functors $v$ and $\mu$ of the Hewitt realcompactification and of the Dieudonné completion, respectively, have been unsuccessful. Usually the assumptions on $X$ and $Y$ are asymmetric (see, for example, the classic papers [9, 22, 23] by Comfort and Ohta). Moreover, Husěk proved in [19] that there is no topological property characterizing the equality $v(X \times Y)=v(X) \times v(Y)$ (and, consequently, the equality $\mu(X \times Y)=\mu(X) \times \mu(Y))$. Thus, the problem of establishing implications, one of whose terms is roughly of the form $v(X \times Y)=v(X) \times v(Y)$ (respectively, $\mu(X \times Y)=\mu(X) \times \mu(Y))$, has attracted very considerable attention.

It is worth noting that the equality $\mu(X \times Y)=\mu(X) \times \mu(Y)$ has a strong relationship with a Pestov-Tkachenko question ([24, 34]): Let $G$ be a topological group, and $\mu G$ the Dieudonné completion of the space $G$. Can the operations in $G$ be extended to $\mu(G)$ in such a way that $\mu(G)$ becomes a topological group containing $G$ as a topological subgroup?

\footnotetext{
*Corresponding Author: Manuel Sanchis: Institut de Matemàtques, Universitat Jaume I de Castelló, E-mail: sanchis@uji.es Research of M. Sanchis was author supported by Ministerio de Economía y Competitividad (Spain) through Project MTM2016-77143-P (AEI/FEDER, UE).

Óscar Valero: Mathematics and Computer Science Department, Balearic Islands University,

E-mail: o.valero@uib.es
} 
Arhangel'skii answered this question in the negative in [2] and he found a relationship between a positive answer to this problem and the distribution of the functor $\mu$ (see also [3, 4, 29, 30]). Arhangel'skii called PTgroups the groups which provide a positive answer to the previous question.

The aim of this paper is to study the relationship between the exponential map and $b_{f}$-continuous extensions of $b_{f}$-continuous functions to $\mu(X) \times Y$. The results applies to the equality $\mu(X \times Y)=\mu(X) \times \mu(Y)$. We generalize several results on the theory by Arhangel'skii [3], Comfort [9], Noble [21] and Ohta [23]. We also obtain a relative version of the classical Glicksberg's theorem characterizing the product of two pseudocompact spaces in the realm of $k_{r}$-spaces. In the last section we present several results on $P T$-groups.

Throughout, all spaces are by default Tychonoff and all topological groups are Hausdorff. A subset $B$ of a space $X$ is said to be bounded (in $X$ ) if each real-valued continuous function on $X$ is bounded on $B$. A space is pseudocompact if it is bounded in itself. Locally pseudocompact spaces are spaces $X$ such that each point of $X$ has a pseudocompact (equivalently, a bounded) neighborhood. We will denote by $b$ the family of all bounded subsets of a space $X$. A function $f$ from a space $X$ into a space $Y$ is said to be $b_{f}$-continuous if the restriction of $f$ to each member of $b$ can be extended to a continuous function on the whole space $X$. A space $X$ is called a $b_{f}$-space if every real-valued $b_{f}$-continuous function on $X$ (equivalently, if every $b_{f}$-continuous function from $X$ into a Tychonoff space $Y$ ) is continuous. Notice that locally pseudocompact spaces and $k_{r^{-}}$ spaces (spaces $X$ where a real-valued function is continuous when its restriction to each compact subset of $X$ is continuous) are examples of $b_{f}$-spaces. Thus, $k$-spaces (spaces $X$ where a subset $A$ is closed if and only if $A \cap K$ is closed in $K$ for every compact subset $K$ of $X$ ), first countable (so metrizable) spaces are $b_{f}$-spaces. It is worth noting that a $\Sigma$-product of an arbitrary product of locally pseudocompact groups and an arbitrary product of locally pseudocompact groups are also examples of $b_{f}$-spaces which are not (in general) $k_{r}$-spaces (for details, see [25, Corollary 4.1, Theorem 4.3]). The image of a $b_{f}$-space under an onto quotient map is a $b_{f^{-}}$ space [25, Lemma 4.2]. Thus, quotients of $b_{f}$-groups are $b_{f}$-spaces [25, Lemma 4.2]. More examples are given in the last section.

Our terminology and notation are standard. For instance, $\mathbb{N}$ means the set of natural numbers, $\mathbb{R}$ the real numbers and $f_{\mid A}$ the restriction of a function $f$ to a subset $A$. We say that a space $X$ is topologically complete (o Dieudonné complete) if $X$ is homeomorphic to a closed subspace of a product of metrizable spaces. It is a well-known fact that for every space $X$ there exists a unique topologically complete space $\mu(X)$, up to homeomorphisms which leave $X$ pointwise fixed, in which $X$ is dense and every continuous function $f$ from $X$ into a topologically complete space $Z$ can be extended to a continuous function on $\mu(X)$. This space is called the Dieudonné completion of $X$. It is clear that a space $X$ is topologically complete if $X=\mu(X)$. It is worth mentioning that, by an outstanding result of Shirota [31], $v X=\mu X$ if the cardinality of every closed discrete subspace of $\mu(X)$ is a non-measurable cardinal number. Thus, our results apply to the Hewitt realcompactification $v(X)$ of $X$ under the assumption that the cardinal of $X$ is non-measurable. For notions and terminology which are not explicitly defined here, the reader might consult [5, 12].

\section{Preliminaries}

Let $F(M, Z)$ denote the set of all real-valued functions from a set $M$ into a set $Z$. Our basic tool will be the so-called exponential map, that is, the natural one-to-one correspondence $\gamma$ between the set $F(X \times Y, Z)$ and $F(X, F(Y, Z))$ which is defined as

$$
\gamma(f)(x)(y)=f(x, y), \quad f \in F(X \times Y, Z) .
$$

The restriction of this map to subspaces will also denoted by $\gamma$. Given two spaces $X$ and $Z, b_{f} C(X, Z)$ stands for the set of all $b_{f}$-continuous functions from $X$ into $Z$. We write $b_{f} C(X)$ when the space $Z$ is the space $\mathbb{R}$ of the real numbers endowed with its usual topology. The exponential map has been used by several authors for studying the relationship $\mu(X \times Y)=\mu(X) \times \mu(Y)$ in the realm of $k$-spaces (see, among others, [9, 19]). We will follow this way in a more general setting, To do this, we take advantage of the following theorem: 
Theorem 2.1. [25, Theorem 3.2] Let $Y$ be a $b_{f}$-space. For each space $X$, the following assertions are equivalent: (i) $\gamma\left(b_{f} C(X \times Y, Z)=b_{f} C\left(X, C_{b}(Y, Z)\right)\right.$ for each topologically complete space $Z$.

(ii) $\operatorname{cl}_{\beta(X \times Y)}(A \times B)=\operatorname{cl}_{\beta(X)}(A) \times \mathrm{cl}_{\beta(Y)}(B)$ whenever $A$ and $B$ are bounded subsets of $X$ and $Y$, respectively.

Note that we can consider condition (ii) as a relative version of the Glicksberg's theorem. So, taking as a stating point this fact, we say that a pair $(X, Y)$ enjoys property (Gl) (in short, $(X, Y)$ is a (Gl) pair) if $X \times Y$ satisfies condition (ii) of Theorem 2.1. By a result of Hernández, Sanchis and Tkachenko [18], the condition (ii) is satisfies if either $X$ or $Y$ is a topological group.

It is a well-known fact that the closure of a bounded subset of a topologically complete space is compact. Therefore, if either $X$ or $Y$ are topologically complete, then $(X, Y)$ is a $(\mathrm{Gl})$ pair. Actually, we have a more general result. We will denote by $\mathfrak{B}$ the Frol'ik's class of all pseudocompact spaces $X$ such that $X \times Y$ is pseudocompact for every pseudocompact space $Y$.

Theorem 2.2. Let $X, Y$ two spaces. If $A \subseteq X$ belongs to $\mathfrak{B}$, then $\operatorname{cl}_{\beta(X \times Y)}(A \times B)=\operatorname{cl}_{\beta(X)}(A) \times \mathrm{cl}_{\beta(Y)}(B)$ for every bounded subset $B$ of $Y$.

Proof. By Corollary 6 in [7], $A \times B$ in bounded in $A \times Y$ and, a posteriori, it is bounded in $X \times Y$. Apply now Theorem 2.8 of [15].

Corollary 2.3. A space $X$ belongs to the class $\mathfrak{B}$ if and only if $(X, Y)$ is a $(\mathrm{Gl})$ pair for every space $Y$.

In the next section we prove that, if $X$ is a $k_{r}$-space, then $(X, Y)$ enjoys property $(\mathrm{Gl})$ for each space $Y$. It seems interesting to remark that the equality $\operatorname{cl}_{\beta(X \times Y)}(A \times B)=\operatorname{cl}_{\beta(X)}(A) \times \mathrm{cl}_{\beta(Y)}(B)$ implies that $A \times B$ is bounded in $X \times Y$. The question of whether the converse is true remains open (see [26, Chapter 4]).

Throughout what follows, we shall freely use without explicit mention the previous facts. More detailed information about the equality $\mathrm{cl}_{\beta(X \times Y)}(A \times B)=\mathrm{cl}_{\beta(X)}(A) \times \mathrm{cl}_{\beta(Y)}(B)$ for bounded subset is available in [14], [27] and in the survey [26, Chapter 4].

\section{The exponential map and $\boldsymbol{b}_{f}$-continuous extensions}

Given two spaces $M$ and $Z, C(M, Z)$ (respectively $C(M)$ ) stands for the family of all continuous functions from $M$ to $Z$ (respectively, from $\mathrm{M}$ to the reals). Let $C_{b}(M, Z)$ denote the space $C(M, Z)$ equipped with the topology of the uniform convergence on bounded subsets. The first result provides an extension theorem for $b_{f}$ continuous functions.

Theorem 3.1. Let $X, Y$ be two $b_{f}$-spaces. If $(X, Y)$ is a $(\mathrm{Gl})$ pair and $Z$ is a topologically complete space, then the following hold:

(i) Every $b_{f}$-continuous function from $X \times Y$ into $Z$ has a $b_{f}$-continuous extension to $\mu(X) \times Y$.

(ii) Every $b_{f}$-continuous function from $X \times Y$ into $Z$ has $a b_{f}$-continuous extension to $X \times \mu(Y)$.

Proof. (i) Let $f$ be a $b_{f}$-continuous function on $X \times Y$. By Theorem 2.1, $\gamma(f)$ belongs to $b_{f} C\left(X, C_{b}(Y, Z)\right)$. Being $X$ a $b_{f}$-space, $\gamma(f)$ is a continuous function. Since $Y$ is a $b_{f}$-space, the function space $C_{b}(Y, Z)$ is Dieudonné complete (see [25, Lemma 3.1]) so that there exists a continuous extension, say $g$, of $\gamma(f)$ to $\mu(X)$.

Consider now the function $\gamma^{-1}(g)$ on $\mu(X) \times Y$. Since $(\mu(X), Y)$ is a (Gl) pair, by Theorem 2.1 again, we have

$$
\gamma\left(b_{f} C(\mu(X) \times Y, Z)\right)=b_{f} C\left(\mu(X), C_{b}(Y, Z)\right)
$$

which implies that $\gamma^{-1}(g)$ belongs to $b_{f} C(\mu(X) \times Y, Z)$. Note that the restriction to $X \times Y$ of $\gamma^{-1}(g)$ coincides with $f$. This completes the proof of (i). The claim (ii) follows in a similar way.

For a $b_{f}$-group $G$ we understand a topological group which is a $b_{f}$-space. 
Corollary 3.2. Let $G$ be $a b_{f}$-group and let $Y$ be $a b_{f}$-space. If $Z$ is a topologically complete space, then the following hold:

(i) Every $b_{f}$-continuous function from $G \times Y$ into $Z$ has $a b_{f}$-continuous extension to $\mu(G) \times Y$.

(ii) Every $b_{f}$-continuous function from $G \times Y$ into $Z$ has $a b_{f}$-continuous extension to $G \times \mu(Y)$.

Observe that the previous results are valid when $Z$ is a metric space (in particular the real line $\mathbb{R}$ ). In fact, Dickinson showed that paracompact spaces are topologically complete [11] and metric spaces are paracompact [33]. As in the previous corollary, being every continuous function $b_{f}$-continuous, Theorem 3.1 enables us to obtain extension theorems for continuous functions when either the space $\mu(G) \times X$ or the space $G \times \mu(X)$ is a $b_{f}$-space. Moreover, our approach allows us to generalize several results obtained in the literature. As a flavor of example, we present several applications.

Corollary 3.3. Let $X$ be a $b_{f}$-space. If $\mu(X) \times Y$ is a $b_{f}$-space and $(X, Y)$ is a (Gl)-pair, then each continuous function on $X \times Y$ into a topologically complete space $Z$ has a continuous extension to $\mu(X) \times Y$.

We need the following result.

Theorem 3.4. [9, Corollary 2.2] If $X$ is a locally compact topological complete space, then $\mu(X \times Y)=X \times \mu(Y)$ for each space $Y$.

Theorem 3.5. If $\mu X$ is locally compact and $Y$ is a $b_{f}$-space such that $(X, Y)$ is a (Gl)-pair, then the equality $\mu(X \times Y)=\mu(X) \times \mu(Y)$ holds.

Proof. Since $\mu(X)$ is locally compact, $X$ is locally pseudocompact and, consequently, a $b_{f}$-space. Moreover, $\mu(X)$ is locally in the class $\mathfrak{B}$ so that, by Corollary 6 in [7], the space $\mu(X) \times Y$ is a $b_{f}$-space. Then, if $Z$ is a topologically complete space, Corollary 3.3 says us that every continuous function on $X \times Y$ into $Z$ has a continuous extension to $\mu(X) \times Y$. Now, by means of Theorem 3.4, it is routine to prove that $\mu(X \times Y)=\mu(X) \times \mu(Y)$ holds.

It is well known that the Dieudonne completion of a locally pseudocompact group $G$ is a locally compact group: in fact, it coincides with the Weil completion of $G$. Then we obtain

Corollary 3.6. If $G$ is a locally pseudocompact group and $X$ is a $b_{f}$-space (in particular, a $b_{f}$-group), then the equality $\mu(G \times X)=\mu(G) \times \mu(X)$ holds.

Comfort and Ross show that the product of two pseudocompact groups is a pseudocompact group [10]. Tkachenko [35] improves this result by proving that every pseudocompact group belongs to $\mathfrak{B}$. According to Glicksberg's theorem, Tkachenko's result is equivalent to the fact that $\beta(G \times X)=\beta(X) \times \beta(Y)$ when $G$ is a pseudocompact group and $X$ is a pseudocompact space. Thus, Corollary 3.6 generalizes both outcomes.

Obviously, Corollary 3.6 holds if locally pseudocompact group is replaced by pseudocompact group. Thus, as a consequence of the previous result, we have

Corollary 3.7. [3, Theorem 5.16] If $G$ is a pseudocompact group and $H$ is a $k$-group, then the equality $\mu(G \times H)=$ $\mu(G) \times \mu(H)$ holds.

Recall that a subset $A$ is bounded in $X$ if every countable locally finite family of pairwise disjoint open sets meeting $A$ is finite. Let $p$ be a free ultrafilter on $\mathbb{N}$ (in symbols, $p \in \omega^{\star}$ ). A subset $A$ of a space $X$ is said to be $p$-bounded (in $\mathrm{X}$ ) if for every sequence $\left\{U_{n}\right\}_{n \in \mathbb{N}}$ of pairwise disjoint open subsets of $X$ meeting $A$, then $\left\{n \in \mathbb{N}: A \cap U_{n} \neq \emptyset\right\} \in p$. Obviously, $p$-bounded subsets are bounded. It is apparent that a compact subset of $X$ is $p$-bounded for every $p \in \omega^{\star}$. For the origins and applications of these concepts in bounded sets theory see [26, Chpater 4]. 
It was showed by Noble [20] that every $k_{r}$-pseudocompact space belongs to the Frolík's class $\mathfrak{B}$. This result was generalizes in [7] where the authors show that if $X$ is a $k_{r}$-space, then, for each space $Y, A \times B$ is bounded in $X \times Y$ for each bounded subset $A$ of $X$ and each bounded subset $B$ of $Y$. By Corollary 4 and Corollary 6 of [7], each locally pseudocompact $k_{r}$-space is locally in the class $\mathfrak{B}$. The following theorem improves these results.

Theorem 3.8. If $X$ is a $k_{r}$-space, then $(X, Y)$ is a (Gl) pair for every space $Y$.

Proof. Let $A$ be a bounded subset of $X$. Consider a sequence $\left\{U_{n}\right\}_{n \in \mathbb{N}}$ of pairwise disjoint open sets meeting $A$. We prove that there is a compact subset of $X$ meeting infinitely many elements of $\left\{U_{n}\right\}_{n \in \mathbb{N}}$. For this, suppose, contrary we claim, that every compact subset of $X$ meets finitely many elements of $\left\{U_{n}\right\}_{n \in \mathbb{N}}$. For each $n \in \mathbb{N}$, choose a point $x_{n} \in U_{n}$.

Next, for each $n \in \mathbb{N}$, let $f_{n}$ be a continuous function on $X$ with $f\left(x_{n}\right)=n$ and $f_{n}\left(X \backslash U_{n}\right)=0$. Define a function $f$ on $X$ as follows:

$$
f(x)=\sum_{n \in \mathbb{N}} f_{n}(x), \quad x \in X .
$$

Notice that, being the elements of the sequence $\left\{U_{n}\right\}_{n \in \mathbb{N}}$ pairwise disjoint, $f$ is well defined. Moreover, by our assumption, $f$ is $k_{r}$-continuous. But $f_{\mid A}$ is unbounded which contradicts that $A$ is bounded in $X$. Thus, there exists a compact subset $K \subseteq X$ and a subsequence $\left\{n_{t}\right\}_{t \in \mathbb{N}}$ such that $K \cap U_{n_{t}} \neq \emptyset$ for every $t \in \mathbb{N}$. Then, since $K$ is $p$-bounded for every $p \in \omega^{\star}$, so also is $A$. The results now follows from [29, Corollary 13].

In the proof of the previous result, the following is implicit.

Corollary 3.9. Each point of a locally pseudocompact $k_{r}$-space has a neighborhood that is $p$-bounded for every $p \in \omega^{\star}$.

Theorem 3.8, Theorem 3.1 and Theorem 3.5 permit us to generalize several results of the literature. The next results improve Theorem 2.7 and Theorem 3.11 of [9] by Comfort and (1) $\Longrightarrow$ (2) of [23] by Ohta.

Corollary 3.10. Assume that both $\mu(X) \times Y$ and $\mu(X) \times \mu(Y)$ are $k_{r}$-space. Then $\mu(X \times Y)=\mu(X) \times \mu(Y)$.

Proof. Let $Z$ be a topologically complete space. Being $Y$ a $k_{r}$-space, $(X, Y)$ is a (Gl) pair by Theorem 3.8. Now Theorem 3.1 says us that each continuous function on $X \times Y$ into $Z$ can be continuously extended to $\mu(X) \times Y$. Let $f$ be a continuous function from $\mu(X) \times Y$ into $Z$. Being $\mu(X)$ topologically complete, Theorem 3.1 applies in order to obtain that $\gamma(f) \in b_{f} C\left(Y, C_{b}(\mu(X), Z)\right.$. Since $\mu(X)$ and $Y$ are $k_{r}$-spaces, an argument similar to the one used in the proof of Theorem 3.1 shows that $f$ has a continuous extension to $\mu(X) \times \mu(Y)$. Thus, $\mu(X \times Y)=$ $\mu(X) \times \mu(Y)$.

Corollary 3.11. If $\mu(X)$ is locally compact and $Y$ is a $k_{r}$-space, then $\mu(X \times Y)=\mu(X) \times \mu(Y)$.

Corollary 3.12. [Compare [23, Theortem 2]] If $X$ is locally pseudocompact and $Y$ is a $k_{r}$-space, then every continuous function $f$ on $X \times Y$ into a topologically complete space $Z$ has a continuous extension to $X \times \mu(Y)$.

Proof. Being $X$ locally pseudocompat, it suffices to prove that $f$ has a continuous extension to $P \times Y$ for each pseudocompact subset $P$ of $X$. By Teorema 3.8 and [7, Theorem 9], $P \times Y$ is a $b_{f}$-space. The result now follows from Theorem 3.8 and Theorem 3.1.

Remark 3.13. In Corollaries 3.10, 3.11 and 3.12 we can replaced $k_{r}$-space by topological group. In fact, as we have commented above, if $G$ is a topological group, then $(G, Y)$ enjoys the property (Gl) for every space $Y$.

Remark 3.14. For a family $\mathfrak{a}$ of bounded subset of a space $X$, the definitions of $\mathfrak{a}_{f}$-space and $\mathfrak{a}_{f}$-continuous function are self-explanatory. Then, if $\mathfrak{b}$ denotes the family of all subsets of a space $X$ that belong to the Frolik's class $\mathfrak{B}$, a natural question is if each $\mathfrak{b}_{f}$-space satisfies Corollaries 3.10, 3.11 and 3.12. 
Perhaps it is appropriate at this point to note that there exists pseudocompact spaces in $\mathfrak{B}$ which are not $p$-pseudocompact (that is, $p$-bounded in itself) for any $p \in \omega^{\star}$ ([28, Example 2.9]). Moreover, a $\mathfrak{b}_{f}$-space need not be pseudocompat: indeed, if $X \in \mathfrak{B}$, consider the disjoint union of countable many copies of $X$ equipped with its natural topology.

However the answer to the previous question is affirmative. To see this, it suffices to use the techniques presented in this paper by applying Theorem 2.2 and the following result. It is possible to obtain it by simply mimicking the proof of Theorem 2.1. All the details are left to the interesting reader.

Theorem 3.15. Let $Y$ be $a \mathfrak{b}_{f}$-space. If $\mathfrak{b} \times b$ stands for the family

$$
\mathfrak{b} \times b=\{A \times B: A \subset X, B \subset Y \text { with } A \in \mathfrak{B}\},
$$

then, for each space $X$, the following holds:

(i) $\gamma\left((\mathfrak{b} \times b)_{f} C(X \times Y, Z)=b_{f} C\left(X, C_{\mathfrak{b}}(Y, Z)\right)\right.$ for each topologically complete space $Z$.

Moreover, if $X$ is a $b_{f}$-space, we have

(ii) $\gamma\left((\mathfrak{b} \times b)_{f} C(X \times Y, Z)=C\left(Y, C_{b}(X, Z)\right)\right.$ for each topologically complete space $Z$.

\section{A note on PT-groups}

In this section we take up a result on $P T$-groups. For this, we turn now a brief discussion of $b_{f}$-hemibounded groups (that is, groups that are $b_{f}$-hemibounded spaces) and Moscow spaces.

Recall that a space $X$ is called hemibounded if there exists a countable family $\left\{B_{n}\right\}_{n \in \mathbb{N}}$ of bounded subsets such that, for every bounded subset $B \subseteq X$, there is a $B_{n}$ such that $B \subseteq B_{n}$. An interesting example of $b_{f^{-}}$ hemibounded group is the free (abelian) topological group $G(X)$ over a pseudocompact space $X$ (see [17]). $b_{f}$-hemibounded spaces appear in a natural way in the theory of function spaces: $C_{b}(X)$ is a Fréchet space if, and only if, $X$ is a $b_{f}$-hemibounded space (see [6]). The definition of a hemicompact space is self-explanatory.

A stimulant result on $b_{f}$-hemibounded spaces is

Theorem 4.1. [8] If $X$ is a $b_{f}$-hemibounded space, then $\mu(X)$ is a $k_{r}$-hemicompact space. Moreover, every compact subset of $\mu(X)$ is contained in the closure (in $\mu(X)$ ) of a bounded subset of $X$.

The notion of a Moscow space was introduced by Arhangels'kii in [1]. A space $X$ is called a Moscow space if $\mathrm{cl}_{X} U$ is the union of $G_{\delta^{-}}$-subsets of $X$ for each open subset $U$ of $X$. Each Moscow group $G$ is a $P T$-group. Actually, $G$ is a strong-PT-group, that is, every real-valued continuous function on $G$ has a continuous extension to the $G_{\delta}$-closure $\rho_{\omega} \mathrm{G}$ of $G$ in its Rajkov completion (see Theorem 1.5, Corollary 1.6 and Theorem 1.7 in [2]). result.

Our aim is to show that $b_{f}$-hemibounded groups are strong-PT groups. First we have the following general

Theorem 4.2. Let $G$ be a topological group. If $\mu(G \times G)=\mu(G) \times \mu(G)$, then $G$ is a PT-group.

Proof. Denote by o the operation on $G$ and consider the function $\alpha: G \times G \rightarrow \mu(G)$ defined as

$$
\alpha\left(g_{1}, g_{2}\right)=g_{1} \circ g_{2}, \quad g_{1}, g_{2} \in G .
$$

Being $\mu(G)$ topologically complete, there is a continuous extension, say $\widetilde{\alpha}$, of the function $\alpha$ to $\mu(G \times G)$. By our hypothesis, $\mu(G \times G)=\mu(G) \times \mu(G)$.

Next we prove that the operation $\widetilde{o}$ defined on $\mu(G)$ as

$$
\begin{array}{cl}
\widetilde{\circ}: \mu(G) \times \mu(G) & \rightarrow \mu(G) \\
\left(h_{1}, h_{1}\right) & \rightarrow \widetilde{\alpha}\left(h_{1}, h_{1}\right)
\end{array}
$$


makes $\mu(G)$ a topological group containing $G$ as a dense subgroup. It is apparent that $\widetilde{o}$ is continuous. First we show that $(\mu(G)$, $\widetilde{o})$ satisfies the associative property. Define the continuous functions $f_{1}, f_{2}: \mu(G) \times \mu(G) \times$ $\mu(G) \rightarrow \mu(G)$ by the rule

$$
f_{1}\left(h_{1}, h_{2}, h_{3}\right)=\widetilde{\alpha}\left(h_{1}, \widetilde{\alpha}\left(h_{2}, h_{3}\right)\right) \text { and } f_{2}\left(h_{1}, h_{2}, h_{3}\right)=\widetilde{\alpha}\left(\widetilde{\alpha}\left(h_{1}, h_{2}\right), h_{3}\right) .
$$

As $G$ is a topological group, $\left.f_{1}\right|_{G \times G \times G}=\left.f_{2}\right|_{G \times G \times G}$. Since $G \times G \times G$ is dense in $\mu(G) \times \mu(G) \times \mu(G)$, we conclude that $f_{1}=f_{2}$, which means exactly that the operation $\widetilde{o}$ is associative.

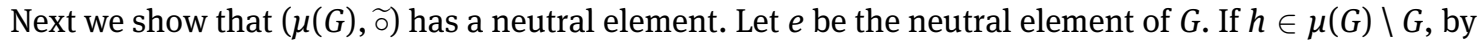
density, there exists a net $\left\{g_{\beta}\right\}_{\beta \in I}$ in $G$ converging to $h$. Since $\widetilde{\alpha}$ is continuous, the net $\left\{g_{\beta}, e\right\}_{\beta \in I}$ converges to $(h, e)$. But, for each $\beta \in I$, we have $g_{\beta}=g_{\beta} \circ e=\widetilde{\alpha}\left(g_{\beta}, e\right)$. Then,

$$
h=\lim _{\beta \in I} g_{\beta}=\lim _{\beta \in I} \widetilde{\alpha}\left(g_{\beta}, e\right)=\widetilde{\alpha}(h, e)=h \widetilde{\circ} e .
$$

In a similar way we can prove that $h=e \widetilde{o} h$. Thus, $e$ is a neutral element of $(G, \widetilde{\circ})$. Now, consider the function $\eta: G \rightarrow \mu(G)$ defined as $\eta(g)=g^{-1}$ for each $g \in G$ with $g^{-1}$ the inverse of $g$. Let $\widetilde{\eta}$ be the continuous extension of $\eta$ to $\mu(G)$. Given $h \in \mu(G) \backslash G$ and a net $\left\{g_{\beta}\right\}_{\beta \in I}$ in $G$ with $\lim _{\beta \in I} g_{\beta}=h$, let $h^{\star}$ the limit of $\left\{\widetilde{\eta}\left(g_{\beta}\right)\right\}_{\beta \in I}$. We show that $e=h \widetilde{\circ} h^{\star}$. Indeed, we have

$$
e=\lim _{\beta \in I}\left(g_{\beta}, g_{\beta}^{-1}\right)=\lim _{\beta \in I} \widetilde{\alpha}\left(g_{\beta}, g_{\beta}^{-1}\right)=\widetilde{\alpha}\left(h, h^{\star}\right)=h \widetilde{\circ} h^{\star} .
$$

An argument similar to the previous one shows that $e=h^{\star} \widetilde{\circ} h$. Thus, $h^{\star}$ is the inverse of $h$. This completes the proof.

Remark 4.3. Topological groups containing a dense abelian group are abelian. So, under the hypothesis of the previous theorem, if $G$ is abelian, so is $\mu(G)$.

If $G$ is a $b_{f}$-hemibounded group and $X$ is a $b_{f}$-hemibounded space, then $\mu(G \times X)=\mu(G) \times \mu(X)$ ([17, Theorem 2.6]). Moreover, $k_{r}$-hemicompact spaces are (normal) $k$-spaces (see Lemma 5.1 of [32])

Theorem 4.4. $b_{f}$-hemibounded groups are Moscou spaces.

Proof. Let $G$ be a $b_{f}$-hemibounded group. By the previous theorem, $\mu(G)$ is a $k$-group (that is, a topological group which is a $k$-space). According to [3, Theorem 2.16], $\mu(G)$ is a Moscou space. Since dense subsets of Moscow spaces are Moscow ([3, Proposition 1.1]), $G$ is Moscow.

Applying Theorem 1.7 of [2], we obtain

Corollary 4.5. If $G$ is $a b_{f}$-hemibounded group, then $G$ is a strong-PT-group.

As a consequence of the previous corollary, we have

Corollary 4.6. The (abelian) free topological group $G(X)$ over a pseudocompact space is a strong-PT group.

The following result is a consequence of Theorem $2.7 \mathrm{in} \mathrm{[17]} \mathrm{and} \mathrm{Theorem} 5.6 \mathrm{in} \mathrm{[3].}$

Theorem 4.7. The product of two $b_{f}$-hemibounded groups is a PT-group. In particular, if $X$ is a pseudocompact space, $G(X) \times G$ is a PT-group for every $b_{f}$-hemibounded group $G$.

\section{References}

[1] Arhangel'skii, A.V. Functional tightness, Q-spaces, and $\tau$-embeddings. Comment. Math. Univ. Carolinae 24:1 (1983), 105120. 
[2] Arhangel'skii, A.V.Moscow spaces, Pestov-Tkačenko Problem, and C-embeddings. Comment. Math. Univ. Carolin. 41 (2000), 585-595.

[3] Arhangel'skii, A.V. Topological groups and C-embeddings. Topology Appl. 115 (2001), 265-289.

[4] Arhangel'skii, A.V. and Hušek, M. Extension of topological and semitopological groups and the product operation. Comment. Math. Univ. Carolin. 42 (2001), 173-186.

[5] Arhangel'skii, A.V. and Tkachenko, M. Topological groups and related structures. Atlantis Studies in Mathematics, 1. Atlantis Press, Paris; World Scientific Publishing Co. Pte. Ltd. Hackensack, NJ, 2008.

[6] Blanchard, N. and Jourlin, M. La topologie de la convergence bornée sur les algébres de fonctions continues. Publ. Dép. Math. (Lyon) 6 (1969), fasc. 2, 85-96 (French).

[7] Blasco, J.L. and Sanchis, M. On the product of two $b_{f}$-spaces. Acta Math. Hung. 62 (1993), 111-118.

[8] Buchwalter, H. Produit topologique, produit tensoriel et c-replétion. Actes du Colloque d'Analyse Fonctionnelle de Bordeaux (Univ. Bordeaux, 1971), pp. 51-71. Bull. Soc. Math. France, Mém. No. 31-32, Soc. Math. France, Paris, 1972 (French).

[9] Comfort,W.W. On the Hewitt realcompactification of a product space. Trans. Amer. Math. Soc. 131 (1968), 107-118.

[10] Comfort, W. W, Ross, K. A. Pseudocompactness and uniform continuity in topological groups. Pacific J. Math. 16 (1966), 483-496.

[11] Dickinson, A. Compactness conditions and uniform structures. Amer. J. Math. 75 (1953), 224-228.

[12] Engelking, R. General Topology. Sigma Series in Pure Mathematics, 6. Heldermann Verlag, Berlin, 1989.

[13] Frolík, Z. The topological product of two pseudocompact spaces. Czechoslovak Math. J. 85 (1960), 339-349.

[14] García-Ferreira, S. and Sanchis, M. On C-compact subsets. Houston J. Math. 23 (1997), 65-86.

[15] García-Ferreira, S., Sanchis, M. and Watson, S. Some remarks on the product of two $C_{\alpha}$-compact subsets. Czechoslovak Math. J. 50 (2000), 249-264.

[16] Glicksberg, I. Stone-Čech compactifications of products. Trans. Amer. Math. Soc. 90 (1959), 369-382.

[17] González, F. and Sanchis, M. Dieudonné completion and bf-group actions. Topology Appl. 153 (2006), 3320-3326.

[18] Hernández, S., Sanchis, M. and Tkacehnko, M. Bounded subsets in spaces and topological groups. Topology Appl. 101, 2000, 21-43.

[19] Hušek,M. Hewitt realcompactification of products. Topics in Topology (Proc. Colloq., Keszthely, 1972), pp. 427-435. Colloq. Math. Soc. János Bolyai, Vol. 8, North-Holland, Amsterdam, 1974.

[20] Noble, N. Countably compact and pseudocompact products. Czechoslovak Math. J. 94 (1969), 390-397.

[21] Noble, N. The continuity of functions on Cartesian products. Trans. Amer. Math. Soc. 149 (1970), 187-198.

[22] Ohta, H. The Hewitt realcompactification of products. Trans. Amer. Math. Soc. 263 (1981), 363-375.

[23] Ohta, H. Local compactness and Hewitt realcompactifications of products. Proc. Amer. Math. Soc. 69 (1978), 339-343.

[24] Pestov, V.G. and Tkavčenko, M.G. Problem 3.28, in Unsolved Problems of Topological Algebra, Academy of Science, Moldova, Kishinev, "Shtiinca?? 1985, p. 18.

[25] Sanchis, M. Continuous functions on locally pseudocompact groups. Topology Appl. 86 (1998), 5-23.

[26] Sanchis, M. Bounded subsets of Tychonoff spaces: a survey of results and problems. Pseudocompact topological spaces, 107-150, Dev.Math. 55, Springer, Cham, 2018.

[27] Sanchis, M. and Tamariz-Mascarúa, A. p-pseudocompactness and related topics in topological spaces. Topology Appl. 98 (1999), 323-343.

[28] Sanchis, M. and Tamariz-Mascarua, A. On quasi-p-bounded subsets. Col l. Math. 80 (1999), 175-189.

[29] Sanchis, M. and Tkachenko, M. Completion of paratopological groups and bounded sets. Monatsh. Math. 183 (2017), 699721.

[30] Sanchis, M. and Tkachenko, M. Dieudonné completion and PT-groups. Appl. Categ. Structures 20 (2012), 1-18.

[31] Shirota, T. A class of topological spaces. Osaka Math. J. 4 (1952), 23-40.

[32] Steven, E. M. and Robert F. W. The strict topology in a completely regular setting: relations to topological measure theory. Can. J. Math., vol. XXIV 5 (1972), 873-890.

[33] Stone, A.H. Paracompactness and product spaces. Bull. Amer. Math. Soc. 54 (1948), 977-982.

[34] Tkačenko, M.G. Subgroups, quotients groups, and products of $\mathbb{R}$-factorizable groups. Topology Proc. 16 (1991), $201-231$.

[35] Tkačenko, M. G. Compactness type properties in topological groups. Czechoslovak Math. J. 38 (113) (1988), 324-341. 